\title{
Cutting the carbon cost of academic travel
}

\author{
John N. Quinton
}

Travel is a key part of academic life, and the carbon emissions associated with it are high. Personal decisions to reduce flying can contribute to climate action, and need not compromise research.

Modern academia is an international game and crossing the globe at 35,000 feet on a long-haul flight to a conference, project meeting or field work is a normal occurrence. The benefits of flying are many-fold: academic collaborations span the globe, and meeting new people, debating and sharing ideas are key to driving science. However, for many academics, the carbon emissions associated with air travel dominate personal carbon budgets, dwarfing other contributions such as from driving or eating a meat-based diet. Those of us dedicating our careers to understanding the pressures on and developing solutions to the threats facing the environment find ourselves with a dilemma.

With the climate crisis upon us, business as usual is not an option. As a first step I explored what I could do to reduce my air travel. With global research collaborations

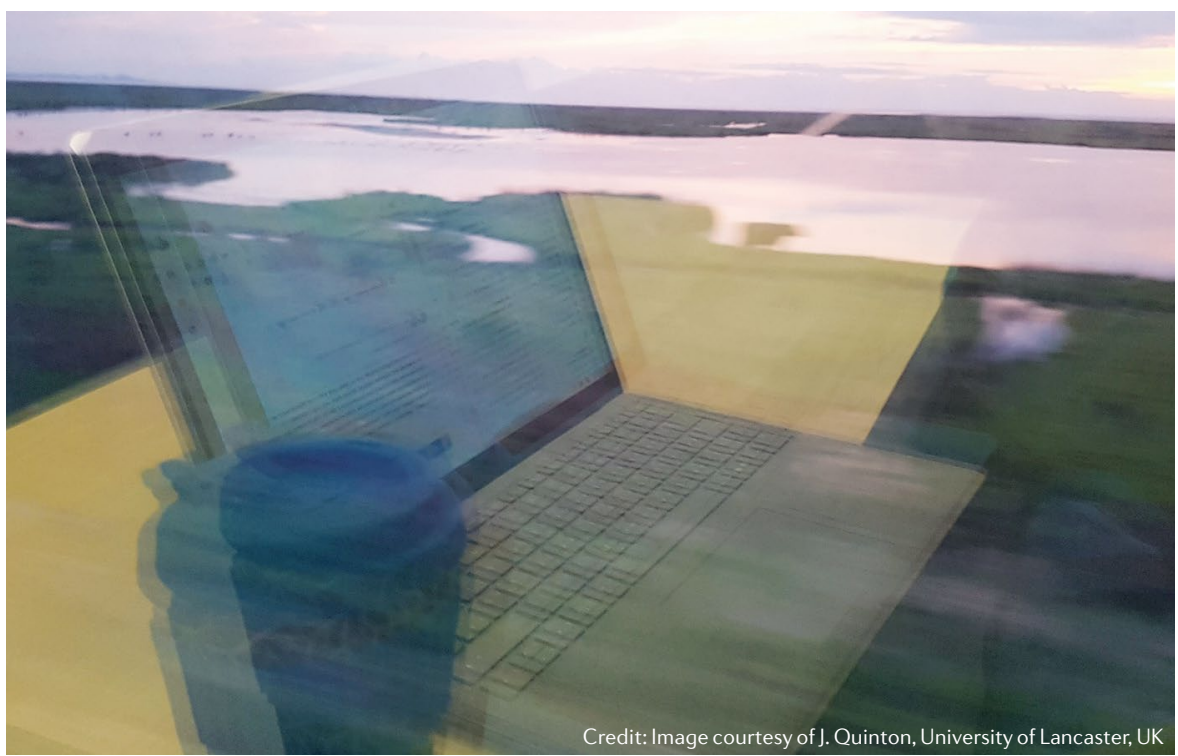

spanning China, South Africa, and Kenya, addressing intercontinental air travel looked difficult. The simplest solution was to replace some of my short-haul flights to Europe which made up $~ 50 \%$ of my travel emissions in 2018 - with train journeys; it took a bit more time, planning and, often, money, but it was possible. While some may think the additional travel time is burdensome, it can be beneficial, offering an opportunity to think, write, relax a bit and, yes, answer emails! I arrive stress-free and feeling a little better about my carbon footprint, plus I have some quality research time along the way - much more productive than the fragmented time associated with short-haul air travel.

Conferences are also recognising this academic movement to decrease flight-related emissions. The EGU, for example, is proactively encouraging 2020 conference goers to use the train, and increasingly train options are being highlighted on conference websites. Social media offers further opportunities for advice and information (for example, \#train2EGU), highlighting the communities' desire to act. It is not only scientific societies that need to get behind academics trying to cut their emissions: the double whammy of more time and money to travel by train means that employers need to get involved too. University travel booking systems could be improved by promoting options that are lowest carbon rather than lowest cost, for example offering the rail alternative for shorthaul destinations and direct flights rather than indirect ones. A fund for overnight stays would help with the extra time required for train travel. The starting point, however, must be for universities and research institutes to measure their academic travel emissions and set targets to reduce them.

We as academics need to take personal responsibility, too. We could start by ensuring we value local and national research as well as international; combining journeys when travelling abroad; taking direct rather than indirect flights; and reaching for the phone or video call rather than the plane. Set a target for yourself to replace at least some of your plane trips with less-carbon-intensive forms of travel and, finally, if you have to travel: offset. I would suggest a donation to charity that seeks to restore degraded peat soils — but as a soil scientist I would say that, wouldn't I?

John N. Quinton

Lancaster Environment Centre, Lancaster University, Lancaster, UK.

e-mail:j.quinton@lancaster.ac.uk https://doi.org/10.1038/s43017-019-0008-3

Competing interests

The author declares no competing interests. 